\title{
COMUNICAÇÃO
}

\section{SENSIBILIDADE DE Colletotrichum gloeosporioides (MANCHA MANTEIGOSA DO CAFEEIRO) A DIFERENTES CONCENTRAÇÕES DE FUNGICIDAS}

\author{
Sensibility of Colletotrichum gloeosporioides (coffee blister spot) \\ to different fungicide concentrations \\ Josimar Batista Ferreira ${ }^{1}$, Mario Sobral de Abreu ${ }^{2}$, Igor Souza Pereira ${ }^{3}$, \\ Katiucia Dias Fernandes ${ }^{4}$, Ricardo Borges Pereira ${ }^{5}$
}

\begin{abstract}
RESUMO
Com o objetivo de avaliar a eficiência de alguns fungicidas sobre Colletotrichum gloeosporioides, agente etiológico da mancha manteigosa do cafeeiro (Coffea arabica L.), testes in vitro foram conduzidos no Laboratório de Diagnose e Controle/UFLA. Utilizouse o método de incorporação de fungicidas ao meio de cultura MEA 2\% para a avaliação da inibição do crescimento micelial e em lâmina escavada contendo água com fungicida para a germinação de conídios. Os fungicidas, tetraconazol, triadimenol, chlorotalonil e mancozeb foram testados quanto à inibição do crescimento do micelial (nas concentrações de 1, 5, 10, 25, 50, 100, 500 e $1.000 \mathrm{mg} \mathrm{L}^{-}$ ${ }^{1}$ ) e quanto à inibição da germinação de conídios (nas concentrações de 1, 5, 10, 25, 50 e $100 \mathrm{mg} \mathrm{L}^{-1}$ ). Os fungicidas tetraconazol e triadimenol apresentaram alta eficiência na inibição do crescimento micelial. Os fungicidas chlorotalonil e mancozeb mostraram baixa eficiência e ineficiência, respectivamente. Quanto à germinação dos conídios, os fungicidas que demonstraram maior eficiência em baixas concentrações foram o chlorotalonil e o tetraconazol.
\end{abstract}

Termos para indexação: Controle in vitro, Antracnose, Coffea arábica.

\begin{abstract}
With the aim of assessing the effect of selected fungicides on Colletotrichum gloeosporioides, the cause of coffee blister spot, in vitro tests were carried out in the Laboratory of Diagnosis and Control/UFLA, Federal University of Lavras, Brazil. In the in vitro experiments the fungicides were incorporated into malt extract medium (MEA 2\%) to evaluate the effect on the fungus growth rate, and concavity slides containing water plus fungicide to assess the conidia germination. The fungicides tetraconazol, triadimenol, chlorothalonil and mancozeb were tested on the mycelial growth inhibition (in the concentrations of 1, 5, 10, 25, 50, 100, 500 and $1.000 \mathrm{mg} \mathrm{L}^{-1}$ ) and on the inhibition conidia germination (in the concentrations of $1,5,10,25,50 \mathrm{e} 100 \mathrm{mg} \mathrm{L}^{-1}$ ). The fungicides tetraconazol and triadimenol showed high efficiency on the mycelial growth inhibition. Chlorotalonil and mancozeb showed low efficiency and inefficiency, respectively. As to the germination of conidia, chlorothalonil and tetraconazol demonstrated to be more efficient in low concentrations.
\end{abstract}

Index terms: In vitro Control, Antracnosis, Coffea arabica.

(Recebido em 21 de maio de 2007 e aprovado em 19 de agosto de 2008)

Diversas são as doenças que provocam danos na cultura do cafeeiro, Coffea arábica L. As de origem fúngica são muito representativas e causam perdas significativas quando não são tomadas medidas de controle adequadas. Embora as principais doenças fúngicas do cafeeiro sejam relativamente bem conhecidas e já se disponham de sistemas de manejo satisfatórios para seu controle, outras doenças representam grande risco para a cafeicultura brasileira. Na maioria das regiões produtoras de Coffea arabica no país, a "mancha manteigosa" do cafeeiro, provocada pelo fungo $C$. gloeosporioides, enquadra-se nessa categoria de risco potencial, em razão do aumento de plantas com sintomas nas lavouras. (Dorizzoto, 1993; Dorizzoto \& Abreu, 1993; Chen, 2002; Nechet \& Abreu,

\footnotetext{
'Engenheiro Agrônomo, Doutor em Fitopatologia - Centro Multidisciplinar/CMULTI - Universidade Federal do Acre/UFAC - Campus Floresta, Estrada Canela Fina, Km 12, Gleba Formoso, Lote 245, Colônia São Francisco - 69980-000 - Cruzerio do Sul, AC josimarferreira@gmail.com ${ }^{2}$ Engenheiro Agrônomo, Doutor em Fitopatologia - Departamento de Fitopatologia/DFP - Universidade Federal de Lavras/UFLA, Cx. P. 3037 - 37200 000 - Lavras, MG - msabreu@ufla.br

${ }^{3}$ Engenheiro Agrônomo, Mestre em Fitopatologia - Departamento de Fitopatologia/DFP - Universidade Federal de Lavras/UFLA - Cx. P. 3037 - 37200 000 - Lavras, MG - igoreloi@yahoo.com.br

${ }^{4}$ Engenheira Agrônoma - Departamento de Fitopatologia/DFP - Universidade Federal de Lavras/UFLA - Cx. P. 3037 -37200-000 - Lavras, MG katiucia@yahoo.com.br

${ }^{5}$ Engenheiro Agrônomo, Doutor em Fitopatologia - Departamento de Fitopatologia/DFP - Universidade Federal de Lavras/UFLA - Cx. P. 3037 -37200000 - Lavras, MG, ricardoborgespereira@yahoo.com.br
} 
2002; Orozco Miranda et al., 2002a,b; Orozco Miranda, 2003).

O patossistema Colletotrichum-cafeeiro ainda é pouco explorado no Brasil e também pouco se conhece sobre o real efeito desse patógeno sobre a cultura. A literatura científica nacional, em diversos trabalhos, caracteriza como saprofítica tal interação, no entanto, trabalhos recentes observam que é sério o problema nas lavouras. Uma vez atacados, os frutos apresentam ligeiras depressões circulares na polpa, de cor castanha e necróticas, as quais vão se alastrando e exibindo lesões necróticas, "típicos cancros", podendo atingir todo o fruto, que fica mumificado. Os sintomas em folhas e ramos novos ocorrem causando seca e necrose, podendo levar à morte das plantas. Neste trabalho, objetivou-se avaliar o efeito in vitro de fungicidas na inibição do crescimento micelial e na germinação de conídios de $C$. gloeosporioides, agente causal da mancha manteigosa.

O presente estudo foi realizado no Laboratório de Diagnose e Controle de Enfermidades Fúngicas em Plantas do Departamento de Fitopatologia (DFP) da Universidade Federal de Lavras (UFLA), Lavras, Minas Gerais. Utilizouse um isolado de $C$. gloeosporioides obtido de haste de plantas de café com mancha manteigosa. Para os bioensaios, este isolado foi crescido em MEA (Blakeslee Malt Extract Autolysate - extrato de malte 3,0\%, peptona $0,1 \%$, glicose $2,0 \%, \mathrm{CuSO}_{4} .5 \mathrm{H}_{2} \mathrm{O} 0,0005 \%, \mathrm{ZnSO}_{4} .7 \mathrm{H}_{2} \mathrm{O}$ $0,001 \%$ e ágar 2,0\%), mantido em câmara de crescimento a $25^{\circ} \mathrm{C} \pm 1$ por 7 dias antes de sua incorporação sobre os meios de cultura contendo os fungicidas.

\section{Avaliação da sensibilidade micelial}

Para este trabalho, foram testados quatro fungicidas descritos na Tabela 1.

No preparo dos meios de cultura com os fungicidas, seguiu-se técnica descrita por Edgington et al. (1971), modificada por Menten et al. (1976). Cada produto utilizado foi dissolvido diretamente em água destilada e esterilizada e, posteriormente, completando seu volume até $100 \mathrm{~mL}$, obtendo-se uma solução estoque de $100.000 \mathrm{mg} \mathrm{L}^{-1}$ do ingrediente ativo. A partir da solução estoque, procedeuse à diluição em série, de tal maneira que cada $\mathrm{mL}$ dessa solução, quando adicionada a $99 \mathrm{~mL}$ de meio MEA fundente, produziu a concentração desejada. Após adicionar o fungicida ao meio de cultura, realizou-se a agitação para homogeneização dos mesmos. No caso do triadimenol, por ter sua formulação granulada, foi colocado sobre agitador magnético por 15 minutos para dissolver os grânulos.

Com o auxílio de um vazador de $0,5 \mathrm{~cm}$ de diâmetro foram retirados discos do meio de cultivo contendo o isolado de C. gloeosporioides, agente causal de mancha manteigosa em cafeeiro, com aproximadamente 7 dias. Esses foram colocados ao centro de placas de Petri de 9 $\mathrm{cm}$ de diâmetro, após solidificação dos meios.

$\mathrm{O}$ delineamento experimental utilizado foi o inteiramente casualizado (DIC), cada tratamento com cinco repetições para cada concentração dos fungicidas. As testemunhas constaram da inoculação de discos miceliais diretamente no meio sem a adição de fungicidas. Os tratamentos foram constituídos pelos fungicidas descritos na tabela 1 , em oito concentrações: 1, 5, 10, 25, 50, 100, 500, $1.000 \mathrm{mg} \mathrm{L}^{-1}$. As placas foram mantidas em câmara de crescimento, à temperatura de $25^{\circ} \mathrm{C} \pm 1$ com fotoperíodo de 12 horas.

Avaliou-se diariamente o crescimento do diâmetro micelial em dois sentidos, perpendicularmente, durante 9 dias após incubação ou até que a testemunha tocasse uma das bordas da placa. $\mathrm{O}$ índice de crescimento micelial (ICM) foi calculado e os dados foram submetidos à análise de variância (pd"0,05) e, quando significativos, à análise de regressão. O ICM foi determinado pela fórmula: $\mathrm{ICM}=\left[\left(\mathrm{C}_{1} /\right.\right.$ $\left.\left.\mathrm{N}_{1}\right)+\left(\mathrm{C}_{2} / \mathrm{N}_{2}\right)+\ldots+\left(\mathrm{C}_{\mathrm{n}} / \mathrm{N}_{\mathrm{n}}\right)\right]$, sendo: $\mathrm{ICM}=$ índice de crescimento micelial; $\mathrm{C}_{1}, \mathrm{C}_{2}, \mathrm{C}_{\mathrm{n}}=$ crescimento micelial do fungo na primeira, segunda e última avaliação; $\mathrm{N}_{1}, \mathrm{~N}_{2}, \mathrm{~N}_{\mathrm{n}}=$ número de dias após a inoculação.

Tabela 1 - Fungicidas utilizados nos bioensaios do controle in vitro de $C$. gloeosporioides.

\begin{tabular}{|c|c|c|c|c|}
\hline Nome comercial & i.a. ${ }^{1}$ & C.i a. ${ }^{2}$ & Modo de ação & Grupo químico \\
\hline Domark & Tetraconazol & $100 \mathrm{gL}^{-1}$ & Inibidor da síntese de ergosterol & Triazol \\
\hline Photon & Triadimenol & $60 \mathrm{gKg}^{-1}$ & Inibidor da síntese de ergosterol & Triazol \\
\hline Bravonil Ultrex & Chlorotalonil & $825 \mathrm{gKg}^{-1}$ & Multissítios & Aromáticos \\
\hline Dithane & Mancozeb & $800 \mathrm{gKg}^{-1}$ & Inibidor de enzimas e proteínas & Ditiocarbamato \\
\hline
\end{tabular}

${ }^{1}$ Ingrediente ativo; ${ }^{2}$ Concentração do ingrediente ativo 
Além disso, foi calculado o $\mathrm{ED}_{50}$ (concentração de ingredientes ativos capazes de inibir $50 \%$ do crescimento micelial) e a concentração mínima inibitória (CMI), ou seja, intervalo entre concentrações dos fungicidas capaz de inibir totalmente o crescimento micelial do fungo, dada pela fórmula: \%inibição $=\{[$ (cresc. da testemunha - cresc. do tratamento)/ cresc. testemunha] x 100\}.

Após o cálculo do $\mathrm{ED}_{50}$, o isolado de $C$. gloeosporioides foi classificado em quatro categorias de sensibilidade, de acordo com a escala de Edgington et al. (1971), em que: $\mathrm{ED}_{50}<1 \mathrm{mg} \mathrm{L}^{-1}$ : alta sensibilidade (AS); $\mathrm{ED}_{50}$ 1-10 $\mathrm{mg} \mathrm{L}^{-1}$ : moderada sensibilidade (MS); $\mathrm{ED}_{50} 10-50 \mathrm{mg}$ $\mathrm{L}^{-1}$ : baixa sensibilidade $(\mathrm{BS}) ; \mathrm{ED}_{50}>50 \mathrm{mg} \mathrm{L}^{-1}$ : insensibilidade (I).

\section{Avaliação da sensibilidade de conídios}

Para avaliar a inibição de germinação dos esporos, utilizou-se o mesmo isolado de $C$. gloeosporioides. A partir de culturas com 7 dias de crescimento, foi obtida uma suspensão de conídios mediante a deposição de $10 \mathrm{~mL}$ de água destilada esterilizada, acrescida de Tween 20 (5 1/10 $\mathrm{mL}$ de água) sobre a superfície da placa de Petri com micélio fúngico, seguido de raspagem das colônias com alça de Drigalski. Em seguida, fez-se a separação do micélio fúngico mais conídios em camada dupla de gaze esterilizada. A concentração conidial foi ajustada para $2 \times 10^{6}$ esporos.mL ${ }^{1}$ com câmara de contagem de Newbawer.

O teste de inibição foi realizado com a diluição dos fungicidas descritos na tabela 1 , em água destilada e esterilizada, obtendo-se as concentrações de 2, 20, 100 e $200 \mathrm{mg} \mathrm{L}^{-1}$. Alíquotas de $30 \mu \mathrm{L}$ da suspensão conidial foram depositadas em lâminas escavadas e, em seguida, $30 \mu \mathrm{L}$ da solução fúngica preparada foram depositados sobre a suspensão de conídios e misturados, obtendo-se as concentrações de $1,5,10,25,50$ e $100 \mathrm{mg} \mathrm{L}^{-1}$ dos fungicidas com uma suspensão conidial de $1 \times 10^{3}$ conídios.mL $\mathrm{mL}^{-1}$. As lâminas escavadas foram mantidas em condições de câmara úmida e temperatura de $25^{\circ} \mathrm{C} \pm 1$, no escuro.

Após as 12 horas de incubação, adicionou-se ácido lático com o objetivo de inibir a germinação dos esporos após esse período. Foram considerados conídios germinados aqueles que apresentaram tubo germinativo com comprimento de, no mínimo, uma vez o tamanho do conídio. O delineamento experimental foi inteiramente casualizado (DIC), com três repetições, em que cada parcela consistiu de três cavidades onde se contou 100 conídios por cavidade, totalizando 300 conídios por parcela. Para análise estatística, obteve-se a porcentagem de conídios germinados.

\section{Sensibilidade micelial}

Os dados referentes ao índice de crescimento micelial e valores médios de percentagem de inibição do isolado de $C$. gloeosporioides em diferentes concentrações de tetraconazol, triadimenol, chlorotalonil e mancozeb são apresentados nas Tabelas 2 e 3.

Para o índice de crescimento micelial (ICM), o fungicida mais eficaz na menor concentração foi o tetraconazol, com o ICM de $2,4 \mathrm{~cm}$, enquanto que o mancozeb, nesta concentração, foi o que apresentou menor eficiência no ICM, com média de $8,0 \mathrm{~cm}$. Já os demais fungicidas (chlorotalonil e triadimenol) apresentaram valores de ICM intermediários, não diferindo estatisticamente a $5 \%$ de probabilidade. No entanto, todos os fungicidas foram eficientes quando comparados à testemunha, que teve um ICM de $8,9 \mathrm{~cm}$, em média. Verificou-se que, com o aumento da concentração, diminuía-se o ICM. De modo geral, todos os fungicidas seguiram tal padrão. Verificou-se que, para os fungicidas tetraconazol e triadimenol, a partir da concentração de 25 e $500 \mathrm{mg} \mathrm{L}^{-1}$, respectivamente, cessavam o crescimento micelial, tendo alta sensibilidade do fungo ao tetraconazol, segundo Edgington et al. (1971) (Tabela 3).

Tabela 2 - Valores médios de percentagem de inibição do crescimento micelial de Colletotrichum gloeosporioides e a concentração mínima inibitória (CMI).

\begin{tabular}{cccccccccc}
\hline \multirow{2}{*}{ Fungicida } & \multicolumn{10}{c}{ Concentrações $\left(\mathrm{mg} \mathrm{L}^{-1}\right)$} \\
\cline { 2 - 9 } & 1 & 5 & 10 & 25 & 50 & 100 & 500 & 1000 & CMI $^{1}$ \\
\hline Mancozeb & $13,0^{2}$ & 21,7 & 23,9 & 32,6 & 34,7 & 43,4 & 60,8 & 71,7 & $>1000$ \\
Chlorotalonil & 20,9 & 27,9 & 51,1 & 58,1 & 67,4 & 74,4 & 88,3 & 90,7 & $>1000$ \\
Tetraconazol & 73,3 & 88,8 & 91,1 & 100,0 & 100,0 & 100,0 & 100,0 & 100,0 & $10-25$ \\
Triadimenol & 25,0 & 54,5 & 52,2 & 65,9 & 77,2 & 95,4 & 100,0 & 100,0 & $100-500$ \\
\hline
\end{tabular}

${ }^{1}$ Intervalo entre concentrações, onde podem-se encontrar valores de $100 \%$ de inibição do crescimento micelial;

${ }^{2}$ Média de cinco repetições. 
A $25 \mathrm{mg} \mathrm{L}^{-1}$, não houve crescimento micelial para o tratamento com o tetraconazol, sendo, portanto, entre os fungicidas testados, o que apresentou menor concentração mínima inibitória (CMI) (Tabela 2). A CMI para o triadimenol ocorreu entre 100 e $500 \mathrm{mg} \mathrm{L}^{-1}$, enquanto que, para os fungicidas mancozeb e chlorotalonil, ocorrem a partir de $1000 \mathrm{mg} \mathrm{L}^{-1}$.

A $1.000 \mathrm{mg} \mathrm{L}^{-1}$, houve crescimento micelial nos tratamentos com mancozeb e chlorotalonil. O ICM do mancozeb foi de $2,6 \mathrm{~cm}$ e do chlorotalonil $0,8 \mathrm{~cm}$, apresentando $71,74 \%$ e $90,70 \%$ de inibição do crescimento micelial. $\mathrm{O}$ tetraconazol foi o fungicida que conduziu ao menor índice de crescimento micelial do patógeno e, apresentou menor $\mathrm{CMI}$, com $\mathrm{ED}_{50}$ menor que $1 \mathrm{mg} \mathrm{L} \mathrm{L}^{-1}$ (Tabela 3).

A eficiência de fungicidas do grupo químico dos triazóis na inibição do crescimento micelial in vitro de Colletotrichum já foi comprovada em outros trabalhos (Freeman et al., 1997). Em testes in vitro com tebuconazole e propiconazole, foi observada inibição do crescimento micelial de $C$. acutatum em baixas concentrações $(<1 \mathrm{mg}$ $\mathrm{L}^{-1}$ ) (Kososki et al., 2001; Tavares, 2004), assim como na inibição do crescimento micelial de $C$. gloeosporioides do mamão (Tavares \& Souza, 2005) e no controle de $C$. gloeosporioides em plantas de citros da variedade Natal por tebuconazole.

Tabela 3 - Efeito dos fungicidas sobre o índice de crescimento micelial e germinação de conídios de $C$. gloeosporioides.

\begin{tabular}{lcccc}
\hline \multirow{2}{*}{ Fungicidas } & \multicolumn{2}{c}{$\begin{array}{c}\text { Sensibilidade } \\
\text { micelial }\end{array}$} & \multicolumn{2}{c}{$\begin{array}{c}\text { Sensibilidade } \\
\text { conídios }\end{array}$} \\
\cline { 2 - 5 } & $\mathrm{ED}_{50}{ }^{1}$ & $\mathrm{E}^{2}$ & $\mathrm{ED}_{50}{ }^{1}$ & $\mathrm{E}^{2}$ \\
\hline Chlorotalonil & 19,75 & $\mathrm{BS}$ & $<1$ & $\mathrm{AS}$ \\
Mancozeb & 266,55 & $\mathrm{I}$ & 11,32 & $\mathrm{BS}$ \\
Tetraconazol & $<1$ & $\mathrm{AS}$ & 5,45 & $\mathrm{MS}$ \\
Triadimenol & 11,4 & $\mathrm{BS}$ & 6,86 & $\mathrm{MS}$ \\
\hline
\end{tabular}

${ }^{1}$ Cálculo da $\mathrm{ED}_{50}$ (concentração suficiente para inibir 50\% do crescimento micelial)

${ }^{2}$ Sensibilidade do fungo aos fungicidas (AS: alta sensibilidade; BS: baixa sensibilidade; MS: moderada sensibilidade e I: insensibilidade).

Dentro do grupo dos triazóis, existe diferença na eficiência do ingrediente ativo, fato este já observado por Tavares \& Souza (2005) para tebuconazole e propiconazole, no crescimento micelial de $C$. gloeosporioides do mamão.
Neste trabalho, o triadimenol, pertencente ao grupo dos triazóis, com $\mathrm{ED}_{50}$ igual a $11,4 \mathrm{mg} \mathrm{L}^{-1}$, foi classificado como de baixa eficiência, assim como o chlorotalonil, com $\mathrm{ED}_{50}$ igual a $19,75 \mathrm{mg} \mathrm{L}^{-1}$ (Tabela 3 ).

A ineficiência do chlorotalonil em testes de inibição do crescimento micelial de C. gloeosporioides in vitro já foi observada por Haddad et al. (2003). Segundo esses autores, os isolados de C. gloeosporioides, causador do mal-das-sete-voltas, não apresentavam completa inibição do crescimento micelial mesmo em altas concentrações (1.000 $\left.\mathrm{mg} \mathrm{L}^{-1}\right)$.

O mancozeb não apresentou resultados satisfatórios nos testes in vitro, sendo classificado como ineficiente $\mathrm{ED}_{50}$ igual a $266,55 \mathrm{mg} \mathrm{L}^{-1}$ (Tabela 4), demonstrando-se também ineficiente no controle da morte de ramos causado por C. gloeosporioides em plantas com mancha manteigosa (Ferreira et al., 2005), apesar de ser utilizado em programas de pulverização em pré-colheita para o controle de antracnose em frutíferas, tais como manga (Fitzell \& Peak, 1984) e mamão (Alvarez \& Nishijima, 1987; Tatagiba et al., 2002). Segundo Kososki et al. (2001), o mancozeb só impediu o crescimento micelial de C. acutatum, da flor-preta-domorangueiro, nas concentrações de 50 e $100 \mathrm{mg} \mathrm{L}^{-1}$, demonstrando, então, sua baixa eficiência nesse tipo de teste.

As curvas de regressão de ICM in vitro para o isolado de C. gloeosporioides, "isolado mancha manteigosa", submetido a diferentes concentrações dos fungicidas testados, encontram-se na figura 1. Foi possível observar que a maioria dos fungicidas testados diminuiu seu ICM quando aumentada a concentração do produto (Figura 1).

As análises de regressões foram realizadas com base na concentração máxima de $100 \mathrm{mg} \mathrm{L}^{-1}$, para todos os fungicidas testados, desconsiderando o tratamento testemunha (Figura 1). O tetraconazol foi o que mais acentuou a redução do ICM, com aumento das concentrações (alta eficiência do produto sobre o fungo), no qual a $1 \mathrm{mg} \mathrm{L}^{-1}$ apresentava redução de 73,33\% do crescimento micelial (Tabela 2). Fungos, principalmente do gênero Colletotrichum, têm demonstrado alta sensibilidade aos fungicidas do grupo dos inibidores da síntese ergosterol. Segundo Tavares (2004) e Tavares \& Souza (2005), fungicidas como imazalil, prochloraz, propiconazole e tebuconazole demonstram-se altamente potente contra $C$. gloeosporioides isolado de mamão, sendo que apenas $1 \mathrm{mg} \mathrm{L}^{-1}$ foi capaz de reduzir, em média, $83,3 \%$ do crescimento micelial do mesmo. 



Figura 1 - Curva de crescimento micelial de C. gloeosporioides, submetido as diferentes concentrações de fungicidas.

\section{Sensibilidade de conídios de $C$. gloeosporioides aos fungicidas}

Os dados referentes à sensibilidade da germinação de conídios de $C$. gloeosporioides são apresentados na tabela 4. O efeito dos fungicidas sobre as taxas de germinação de conídios foi diferente dos observados para o crescimento micelial, resultando em diferentes processos biológicos mensurados a partir destas variáveis.

Em consideração à inibição da germinação, o chlorotalonil foi o fungicida que apresentou melhor desempenho em todas as concentrações (Figura 2). A 1 $\mathrm{mg} \mathrm{L}^{-1}$, a germinação de conídios foi de $16,66 \%$, enquanto que o tetraconazol, considerado o mais eficiente na inibição do crescimento micelial, teve germinação de $62,33 \%$ nesta mesma concentração (Tabela 4). O chlorotalonil apresentou um $\mathrm{ED}_{50}$ menor que $1 \mathrm{mg} \mathrm{L}^{-1}$, demonstrando, assim, sua alta eficiência na inibição de germinação de esporos, sendo classificado como um fungicida de alta sensibilidade, segundo os critérios de Edgington et al. (1971) (Tabela 3). Isso, provavelmente ocorreu pelo fato de esse fungicida agir diretamente na germinação de esporos. Esse resultado é semelhante ao encontrado por Kososki et al. (2001), para a germinação de C. acutatum da flor-preta-do-morangueiro, porém, diverge do resultado obtido por Tavares \& Souza (2005) em que, a $1 \mathrm{mg} \mathrm{L}^{-1}$ ocorria germinação de 70,4\% dos conídios de $C$. gloeosporioides do mamão e, a $10 \mathrm{mg} \mathrm{L}^{-1}$, a germinação era totalmente inibida. No presente trabalho, a inibição total da germinação de conídios para o chlorotalonil ocorreu entre 50 e $100 \mathrm{mg} \mathrm{L}^{-1}$ (Tabela 4).

Observou-se que, com o aumento da concentração dos fungicidas, diminuía o percentual da germinação dos conídios (Figura 2), fato mais evidente para o chlorotalonil (Tabela 4). Na concentração de $100 \mathrm{mg} \mathrm{L}^{-1}$, a maioria dos fungicidas inibiu por completo a germinação, exceto o triadimenol, com germinação de 13,0\%, considerado como o fungicida que menos reduziu a germinação para todas as concentrações testadas (Tabela 4).

Os fungicidas tetraconazol e triadimenol apresentaram $\mathrm{ED}_{50}$ igual a 5,45 e $6,86 \mathrm{mg} \mathrm{L}^{-1}$, respectivamente, tendo moderada sensibilidade na inibição da germinação de conídios. Já o mancozeb apresentou $\mathrm{ED}_{50}$ $11,32 \mathrm{mg} \mathrm{L}^{-1}$, demonstrando baixa sensibilidade na inibição da germinação. Assim como no teste do índice do crescimento micelial (ICM), o mancozeb foi o fungicida de menor eficiência para inibir a germinação de esporos (Tabela 3), corroborando, mais uma vez, os resultados preliminares apresentados por Ferreira et al. (2005). 
Tabela 4 - Valores médios de percentagem da germinação de conídios de Colletotrichum gloeosporioides, em diferentes concentrações de diversos grupos de fungicidas.

\begin{tabular}{ccccc}
\hline \multirow{2}{*}{ Concentração $\left(\mathrm{mg} \mathrm{L}^{-1}\right)$} & \multicolumn{4}{c}{ Fungicidas } \\
\cline { 2 - 5 } & Mancozeb & Chlorotalonil & Tetraconazol & Triadimenol \\
\hline 0 & $82,00 \mathrm{a}$ & $87,00 \mathrm{a}$ & $86,66 \mathrm{a}$ & $85,33 \mathrm{a}$ \\
1 & $70,66 \mathrm{c}$ & $16,66 \mathrm{a}$ & $62,33 \mathrm{bc}$ & $52,66 \mathrm{~b}$ \\
5 & $76,00 \mathrm{c}$ & $12,33 \mathrm{a}$ & $49,66 \mathrm{~b}$ & $59,33 \mathrm{~b}$ \\
10 & $40,33 \mathrm{bc}$ & $5,00 \mathrm{a}$ & $38,00 \mathrm{~b}$ & $49,66 \mathrm{c}$ \\
25 & $20,66 \mathrm{~b}$ & $3,33 \mathrm{a}$ & $21,00 \mathrm{~b}$ & $20,66 \mathrm{~b}$ \\
50 & $6,33 \mathrm{ab}$ & $1,00 \mathrm{a}$ & $0,66 \mathrm{a}$ & $14,00 \mathrm{~b}$ \\
100 & $0,00 \mathrm{a}$ & $0,00 \mathrm{a}$ & $0,00 \mathrm{a}$ & $13,00 \mathrm{~b}$ \\
\hline
\end{tabular}

*Médias seguidas de mesma letra nas linhas não diferem entre si, pelo teste de Tukey a $0,5 \%$ de probabilidade.

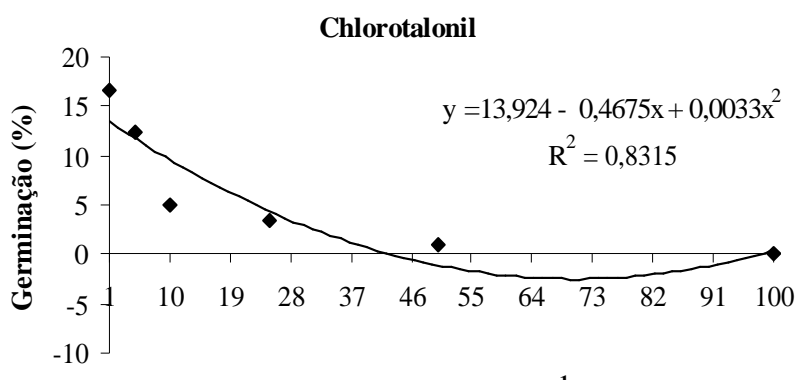

Concentrações $\left(\mathrm{mg} \mathrm{L}^{-1}\right)$

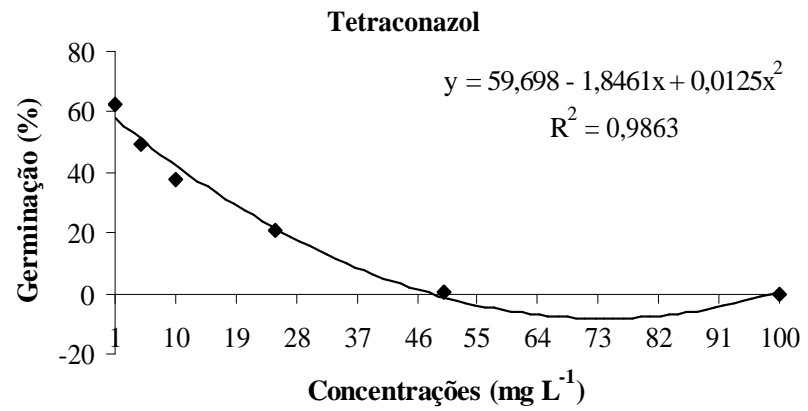



Triadimenol

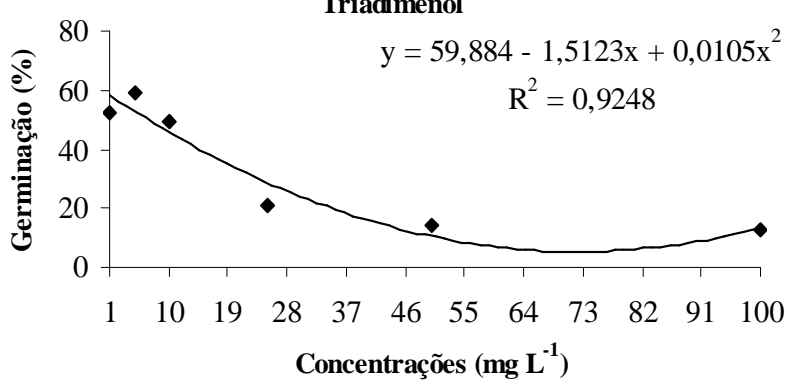

Figura 2 - Curva de germinação de C. gloeosporioides, submetido às diferentes concentrações de fungicidas.

O tetraconazol foi o fungicida que determinou o menor índice de crescimento micelial de $C$. gloeosporioides isolado de lesões de mancha manteigosa em cafeeiro, além da menor concentração mínima inibitória e $\mathrm{ED}_{50}$ menor que $1 \mathrm{mg} \mathrm{L}^{-1}$. O mancozeb não apresentou resultados satisfatórios nos testes in vitro. Já o chlorotalonil, em relação à inibição da germinação, foi o fungicida que apresentou melhor desempenho, enquanto que, os fungicidas mancozeb e triadimenol apresentaram baixa eficiência na inibição da germinação de $C$. gloeosporioides.

\section{REFERÊNCIAS BIBLIOGRÁFICAS}

ALVAREZ, A.M.; NISHIJIMA, W.T. Post harvest diseases of papaya. Plant Disease, Saint Paul, v.71, n.8, p.681-686, Aug. 1987. 
CHEN, Z. Morphocultural and pathogenic comparisons between Colletotrichum kahawae and Colletotrichum gloeosporioides isolated from coffee berries. 2002. 163p. Tese (Doutorado em Engenharia Agronômica)Universidade Técnica de Lisboa, Lisboa, 2002.

DORIZZOTTO, A. Caracterização morfológica e patogenicidade de Colletotrichum sp. associados a cafeeiros (Coffea arabica $\mathbf{L}$.) em dois municípios de Minas Gerais. 1993. 67p. Dissertação (Mestrado em Fitopatologia)-Universidade Federal de Lavras, Lavras, 1993.

DORIZZOTO, A.; ABREU, M.S. Caracterização cultural e morfológica de Colletotrichum coffeanum NOAK e Colletotrichum gloeosporioides PENZ. Fitopatologia Brasileira, Brasília, v.18, ago. 1993. Resumo.

Suplemento.

EDGINGTON, L.V.; KHEW, K.L.; BARRON, G.L. Fungitoxic spectrum of benzimidazole compounds. Phytopathology, Saint Paul, v.61, n.1, p.42-44, Jan. 1971.

FERREIRA, J.B.; SILVA, E.H.; FERNANDES, K.D.; PEREIRA, R.B.; ABREU, M.S.; PEREIRA, I.S. Efeito de fungicidas no controle da seca de ramos do cafeeiro $(C$. arabica L.) com mancha manteigosa (Colletotrichum spp.). Fitopatologia Brasileira, Brasília, v.30, p.111-111, ago. 2005. Resumo. Suplemento.

FITZELL, R.D.; PEAK, C.M. The epidemiology of anthracnose disease of mango: inoculum sources, spore production and disposal. Annals of Applied Biology, Warwick, v.104, n.1, p.53-59, Jan. 1984.

FREEMAN, S.; NIZANI, F.; DOTAN, S.; EVEN, S.; SANDO, T. Control of Colletotrichum acutatum in strawberry under laboratory, greenhouse and field conditions. Plant Disease, Saint Paul, v.81, n.7, p.749752, July 1997.

HADDAD, F.; MAFFIA, L.A.; MIZUBUTI, E.S.G. Avaliação de fungicidas para o controle de Colletotrichum gloeosporioides em cebola. Fitopatologia Brasileira, Brasília, v.28, n.4, p.435-437, jul./ago. 2003.

KOSOSKI, R.M.; FURLANETTO, C.; TOMITA, C.K. Efeito de Fungicidas em Colletotrichum acutatum e
Controle da Antracnose do Morangueiro. Fitopatologia Brasileira, Brasília, jul. 2001. Resumo.

MENTEN, J.O.M. et al. Efeito de alguns fungicidas no crescimento micelial da Macrophomina phaseolina in vitro. Fitopatologia Brasileira, Brasília, v.1, p.57-66, fev. 1976.

NECHET, K.L.; ABREU, M.S. Caracterização morfológica e testes de patogenicidade de isolados de Colletotrichum sp. obtidos de cafeeiro. Ciência e Agrotecnologia, Lavras, v.26, n.6, p.1135-1142, nov./dez. 2002.

OROZCO MIRANDA, E.F. Caracterização morfológica, molecular, bioquímica e patogênica de isolados de Colletotrichum spp. associados ao cafeeiro em Minas Gerais e Comparação com Colletotrichum kahawae. 2003. 147p. Tese (Doutorado em Fitopatologia)Universidade Federal de Lavras, Lavras, 2003.

OROZCO MIRANDA, E.F.; FREITAS, M.; PIGOZZO, P.; ABREU, M.S. Estudo da transmissão de Colletotrichum spp. por sementes de café arabica (Coffea arabica). In: CONGRESSO DE PÓS-GRADUAÇÃO, 11., 2002, Lavras, MG. Resumos... Lavras: UFLA/APG, 2002a.

OROZCO MIRANDA, E.F.; FREITAS, M.; PIGOZZO, P.; ABREU, M.S. Incidência de Colletotrichum spp. em frutos cereja e sementes de café arábica (Coffea arabica) no estado de Minas Gerais. In: CONGRESSO DE PÓSGRADUAÇÃO, 11., 2002, Lavras, MG. Resumos... Lavras: UFLA/APG, 2002b.

TATAGIBA, J.S.; LIBERATO, J.R.; ZAMBOLIM, L.; VENTURA, J.A.; COSTA, H. Controle e condições climáticas favoráveis à antracnose do mamoeiro. Fitopatologia Brasileira, Brasília, v.27, p.186-192, mar./ abr. 2002.

TAVARES, G.M. Controle químico e hidrotérmico da antracnose em frutos de mamoeiro (Carica papaya $\mathrm{L}$.) na pós-colheita. 2004. 55p. Dissertação (Mestrado em Fitopatologia)-Universidade Federal de Lavras, Lavras, 2004.

TAVARES, G.M.; SOUZA, P.E. Efeito de fungicidas no controle in vitro de Colletotrichum gloeosporioides, agente etiológico da Antracnose do Mamoeiro (Carica papaya 1.). Ciência e Agrotecnologia, Lavras, v.29, n.1, p.52-59, jan./fev. 2005. 